\title{
Genome-wide association study of rice genes and loci conferring resistance to Magnaporthe oryzae isolates from Taiwan
}

\author{
Heng-An Lin ${ }^{1 \dagger}$, Szu-Yu Chen ${ }^{1 \dagger}$, Fang-Yu Chang ${ }^{2}$, Chih-Wei Tung ${ }^{3}$, Yi-Chia Chen ${ }^{1}$, Wei-Chiang Shen ${ }^{1}$, \\ Ruey-Shyang Chen ${ }^{4}$, Chih-Wen $\mathrm{Wu}^{2}$ and Chia-Lin Chung ${ }^{1 *}$
}

\begin{abstract}
Background: Rice blast, caused by Magnaporthe oryzae, is an important rice disease occurring in all rice-growing areas. To manage blast disease effectively and in an environmentally friendly way, it is important to continually discover diverse resistant resources for breeding. In this study, genome-wide association study (GWAS) was used to map genes/loci resistant to rice blast in the open-access rice diversity panel 1 (RDP1), previously genotyped with a 44K single-nucleotide polymorphism array. Two geographically and genetically different $M$. oryzae isolates from Taiwan, D41-2 and 12YL-DL3-2, were used to challenge RDP1. Infected leaves were visually rated for lesion type (LT) and evaluated for proportion of diseased leaf area (\%DLA) by image analysis software.

Results: A total of 32 quantitative trait loci (QTLs) were identified, including 6 from LT, 30 from DLA, and 4 from both LT and DLA. In all, 22 regions co-localized with previously reported resistance $(R)$ genes and/or QTLs, including two cloned $R$ genes, Pita and Ptr; 19 mapped $R$ loci, and 20 QTLs. We identified 100 candidate genes encoding leucine-rich repeat-containing proteins, transcription factors, ubiquitination-related proteins, and peroxidases, among others, in the QTL intervals. Putative resistance and susceptibility haplotypes of the 32 QTL regions for each tested rice accessions were also determined.
\end{abstract}

Conclusions: By using Taiwanese M. oryzae isolates and image-based phenotyping for detailed GWAS, this study offers insights into the genetics underlying the natural variation of blast resistance in RDP1. The results can help facilitate the selection of desirable donors for gene/QTL validation and blast resistance breeding.

Keywords: Rice blast, Quantitative trait loci (QTLs), Genome-wide association study (GWAS), Haplotype analysis, Rice diversity panel 1 (RDP1)

\section{Background}

Rice is considered the major staple food of over half of the world's population. In 2014-2015, the global rice yield was 494.7 million tonnes (milled basis) and the cultivated area 162 million hectares, mostly in Asia (Food and Agriculture Organization of the United Nations 2016). Rice blast is a devastating disease occurring in all rice cultivated areas. This polycyclic disease

\footnotetext{
*Correspondence: clchung@ntu.edu.tw

${ }^{+} \mathrm{Heng}$-An Lin and Szu-Yu Chen contributed equally to this work 1 Department of Plant Pathology and Microbiology, National Taiwan University, No. 1, Sec. 4, Roosevelt Rd., Taipei 10617, Taiwan Full list of author information is available at the end of the article
}

is caused by the filamentous ascomycete fungus Magnaporthe oryzae (anamorph Pyricularia oryzae), which can infect all parts of the rice plant at all growth stages (Wilson and Talbot 2009). Blast disease can be managed well with fungicides (e.g., isoprothiolane, thiophanatemethyl) and antibiotics (e.g., kasugamycin) applied at the proper time. However, abuse of agricultural chemicals can cause additional costs and undesirable environmental side effects. An economic and eco-friendly alternative is to use resistant rice varieties. However, owing to the rapid evolution of pathogen avirulence $(A v r)$ genes in field populations of $M$. oryzae, resistant varieties can be overcome in a few years after 
their release and large-scale cultivation (Chen et al. 2009; Zeigler et al. 1994). To more effectively control rice blast, it is important to continually discover new resistant-related genes for breeding.

Both qualitative and quantitative types of resistance against rice blast have been reported. To date, more than 500 quantitative trait loci (QTLs) have been identified in $~ 30$ different studies, mostly using populations derived from indica/japonica crosses (Ashkani et al. 2016; Miah et al. 2013; Sharma et al. 2012). At least 100 resistance $(R)$ genes have been mapped in the rice genome. Most $R$ genes are from japonica and indica lines and $4 \%$ are from wild species (Sharma et al. 2012). A total of $31 R$ genes (Pi1, Pi2/Pi9/Pi50/Pigm/Piz-t, Pi5, Pi21, Pi25/ Pid3, Pi35/Pish, Pi36, Pi37, Pi54rh, Pi56, Pi63, Pi64, Pia/PiCO39, Pib, Pid2, Pi-k/Pik-m/Pik-p, Pik-h/Pi54, Pit, Pita, NLS1 and Pb1) have been cloned and characterized (Ashkani et al. 2016; Das et al. 2012; Deng et al. 2009; Fukuoka et al. 2014; Ma et al. 2015; RiceData 2012; Tang et al. 2011). Most blast resistance genes encode nucleotide binding site-leucine-rich repeat (NBS-LRR) proteins (Koide et al. 2009; McDowell and Woffenden 2003). The NBS domain functions in signaling, and the LRR domain usually plays an important role in R-AVR protein-protein interaction (Marone et al. 2013). There are three non-NBS-LRR blast $R$ genes: Pid2, encoding a B-lectin kinase protein (Chen et al. 2006); pi21, encoding a proline-rich protein (Fukuoka et al. 2009); and Ptr, encoding two isoforms each with four Armadillo repeats (Zhao et al. 2018).

The success of molecular breeding for developing new resistant varieties for blast management depends on finescale localization of $R$ genes and QTLs. The conventional approach to mapping QTLs is linkage analysis, which involves an experimental population of $\mathrm{F}_{2}$, back-crossed, double haploid, or recombinant inbred lines derived from two parental lines with contrasting phenotypes (Collard et al. 2005). Linkage analysis with a bi-parental population is ideal for low-resolution mapping and allows for testing a maximum of two alleles at a locus. Another mapping approach, genome-wide association study (GWAS), is used to correlate patterns of genomic variation with phenotype(s) in a collection of diverse genotypes (Buckler et al. 2009; Huang and Han 2014). Because of the numerous historic recombination events resulting from a long natural evolution (Soto-Cerda and Cloutier 2012), GWAS can identify QTLs at high resolution, possibly to the gene level, and does not require the time and labor needed for constructing a mapping population (Oraguzie et al. 2007; Pasam and Sharma 2014). With the development of high-throughput genotyping techniques, GWAS has also been used to dissect complex traits in crops such as rice, maize, and wheat (Buckler et al. 2009;
Huang et al. 2010; Huang and Han 2014; Rasheed et al. 2014).

Several populations of rice have been established to investigate traits related to agronomic properties and stress tolerance by GWAS (Begum et al. 2015; Famoso et al. 2011; Huang et al. 2010, 2012; Norton et al. 2014; Wang et al. 2014; Zhao et al. 2011). Among those, Wang et al. (2014) inoculated a subset of 366 indica rice accessions from a population of 517 China landraces with 16 local M. oryzae strains and detected 30 blast-associated loci and several candidate genes (Wang et al. 2014). Another established population, rice diversity panel 1 (RDP1), is a collection of 421 rice accessions containing landraces and cultivars from 82 countries (Eizenga et al. 2014). The open-access RDP1 was genotyped with 44,100 single nucleotide polymorphisms (SNPs) and used to discover loci associated with agronomic traits (Zhao et al. 2011); aluminum tolerance (Famoso et al. 2011); grain concentrations of arsenic, copper, molybdenum, and zinc (Norton et al. 2014); and blast resistance (Kang et al. 2016; Mgonja et al. 2016; Zhao et al. 2011). Recently, a panel of 1568 diverse rice accessions (RDP1 included) and high-density 700,000 SNP genotyping data were released to the rice community (McCouch et al. 2016).

The current research aimed to use GWAS to search for blast resistance genes and QTLs in RDP1. RDP1 has been used to locate blast resistance loci in several previous studies. A major QTL was identified with 413 accessions by using a mixture of three $M$. oryzae isolates from the United States (Zhao et al. 2011); 97 QTLs were mapped with 390 accessions by using five M. oryzae isolates from South Korea, China, Columbia, India, and the Philippines (Kang et al. 2016); 31 QTLs were mapped with 162 accessions by using four M. oryzae isolates from Tanzania, Uganda, Kenya, and Burkina Faso (Mgonja et al. 2016); and 16 QTLs were mapped with 413 accessions by using natural infection in three blast nurseries in Shanghang, Wuchang, and Taojiang in China (Zhu et al. 2016). Here we selected two Taiwan isolates to challenge the RDP1 accessions. Image-based phenotyping and detailed GWAS offered more insights into the genetics underlying the natural variation of blast resistance in RDP1. Analysis of haplotypes helped identify rice accessions with favorable alleles at candidate QTL regions. The results can help facilitate marker development and the selection of desirable donors for blast resistance breeding.

\section{Methods}

\section{Plant and fungal materials}

Seeds of RDP1 were acquired from the Genetics Stocks Oryza (GSOR) germplasm collection (Agricultural Research Service, US Department of Agriculture). Sufficient seeds from 314 of 421 diverse accessions were 
successfully reproduced in a greenhouse at Kaohsiung District Agriculture Research and Extension Station and in the Phytotron at National Taiwan University during 2011-2013. The 314 accessions contained 55 indica, 51 aus, 83 temperate japonica (TEJ), 75 tropical japonica (TRJ), 5 aromatic, and 45 admixed varieties (Additional file 1: Table S1). Principle component analysis (PCA) defined the five subpopulations indica, aus, TEJ, TRJ, and aromatic according to clear clustering based on the top four principal components (PCs) explaining $50 \%$ of the genetic variation in RDP1, and the admixed accession did not fit well into any clusters (Zhao et al. 2011). Because aus is closely related to indica and aromatic is related to japonica (Kovach et al. 2007), the full population could be further divided into indica (indica and aus) and japonica (TEJ, TRJ, and aromatic) varietal subgroups. Two susceptible varieties, LTH and Lomello, were included as positive controls.

From the collection of nationwide surveys of $M$. oryzae isolates in Taiwan during 2009-2013 (Shen, W.-C. and Chen, R.-S, unpublished), two geographically and genetically different isolates, D41-2 and 12YL-DL3-2, were chosen for blast inoculation. D41-2, isolated from Chiayi in 2009, by Chen, belongs to a dominant Pot 2 fingerprint lineage in Taiwan; 12YL-DL3-2 was isolated from Yilan in 2012, by Shen, belongs to a minor Pot2 lineage. Both isolates grow and sporulate well on artificial media and showed high virulence in LTH and Lomello. D41-2 and 12YL-DL3-2 were inoculated in 8 international standard blast differential varieties and 16 Taiwan blast differential varieties. The reaction patterns indicate their difference in pathogenicity (Additional file 2: Table S2).

\section{Evaluation of blast resistance}

Resistance of rice accessions to D41-2 and 12YL-DL3-2 was evaluated in three and two independent inoculation trials, respectively, with two replications per trial and 6-7 seedlings per rice accession per replication. Inoculations with D41-2 and 12YL-DL3-2 were conducted from December 2013 to March 2014 and February to July 2015, respectively. Inoculation followed methods modified from Azizi et al. (2015) and Valent et al. (1991). M. oryzae was cultured on oat meal agar (OMA) for 2 weeks at $26{ }^{\circ} \mathrm{C}$ in a $12 / 12$-h light/dark photoperiod in a growth chamber. Conidia were dislodged by using a glass rod and $0.05 \%$ Tween 20 (Sigma, USA), filtered through sterilized double-layered cheesecloth, and adjusted to $2 \times 10^{5}$ conidia/ml by using a haemocytometer. Rice seeds were sown in plug trays ( 5 seeds per plug). In each tray, 48 rice accessions (2 plugs each) and 2 susceptible control varieties (4 plugs each) were arranged in a randomized complete block design (RCBD). Rice seedlings were grown at $28 / 26{ }^{\circ} \mathrm{C}$ day/night temperature and $16 / 8$-h light/ dark photoperiod (luminous intensity 7000-8000 lx) in a growth chamber. Seedlings were fertilized with a 500X dilution of HYPONeX No.5 (N:P:K=30:10:10) (HYPONEX Corp., USA) at 7 and 20 days after planting. Three- to four-leaf-stage seedlings were inoculated by spraying with $2 \times 10^{5}$ conidia $/ \mathrm{ml}$ suspension $(50 \mathrm{ml}$ per tray) with use of an airbrush (Ming Yang, Taiwan) at $10-15$ psi. Inoculated seedlings were maintained at $26{ }^{\circ} \mathrm{C}$ and $95-100 \%$ relative humidity in a sealed plastic storage box, with its interior covered with wet paper towels. After $36 \mathrm{~h}$ of incubation, the seedlings were grown at $28 / 26^{\circ} \mathrm{C}$ day/night temperature and 16/8-h light/dark photoperiod (7000-8000 lx) for disease development. Seven days after inoculation, the second or third leaves were excised, placed on a light box (Chartmat, Taiwan), flattened with a transparent slide and photographed (Canon EOS 700D, Japan; ISO: 400, F8.0, shutter rate 1/100). Each digital image contained 3-7 diseased leaves from one rice accession in a replication. Diseased leaf area (DLA) was analyzed by using Assess 2.0 (Lamari 2008), with the color threshold manually adjusted to correctly differentiate lesions from healthy tissue. Predominant lesion type (LT) was visually rated according to the Standard Evaluation System for Rice (IRRI 2014), with scores 0, 1, and 3 considered resistant (R) and 5, 7, and 9 considered susceptible (S). Only rice accessions showing consistent results in all replications were included for subsequent analyses. Pearson correlation analysis was used to analyze the correlation between DLA and LT by using SAS 6.1 (SAS Inst. Inc., Cary, NC, USA).

\section{GWAS}

The GWAS involved use of the 44K SNP dataset $(36,775$ high-quality SNPs) (Zhao et al. 2011). The physical map positions of the SNPs were converted from the MSU v6.0 Nipponbare rice reference genome (MSU6) to the MSU v7.0 Nipponbare rice reference genome (MSU7) by using the assembly converter tool in Gramene (http://www. gramene.org/). The phenotypic datasets were LT and DLA. The LT scores were averaged over different replications and trials. Accessions showing resistant-type lesions (LT in each trial $=0,1,3$ ) and susceptible-type lesions (LT in each trial $=5,7,9$ ) were defined as R and $\mathrm{S}$ accessions, respectively. To control the variation among blocks in different inoculation experiments, best linear unbiased estimates (BLUEs) for DLA data were generated by using TASSEL 5.0.5 (Bradbury et al. 2007).

The generalized linear model (GLM) and mixed linear model (MLM) were used for analyzing different traits (LT, DLA) and populations (full population, indica, japonica) by using TASSEL 5.0.5. The formulas for the GLM and MLM were $y=X \beta+e$ and $y=X \beta+Z u+e$, respectively, where $y$ is the vector of phenotypic data, $\beta$ is the vector 
of genotypic data and population structure (Q), $u$ is the vector estimated from kinship matrix (K), $e$ is vector of residuals, $X$ is the matrix of genotype and population structure, and $Z$ is the known design matrices (Bradbury et al. 2007). The population structure was obtained by PCA with TASSEL 5.0.5. We tested GLM and MLM with/without the population structure as covariates (GLM, GLM-Q, MLM-K, MLM-K+Q). Quantile-quantile (Q-Q) plots were produced by using TASSEL 5.0.5 to assess the fitness of different models for each phenotype dataset. Q-Q plots showing less deviation from the $y$ (observed test statistics) $=\mathrm{x}$ (expected test statistics) lines suggest less systemic bias (Reed et al. 2015). Manhattan plots were generated by using the qqman package in $R(R$ Development Core Team 2011).

To understand the linkage disequilibrium (LD) structure, pairwise LD analysis of SNPs involved using TASSEL 5.0.5, and LD blocks were defined by using Haploview (Barrett et al. 2005) with default settings. The LD blocks containing more than three SNPs with $P<3.1 \times 10^{-4}\left[-\log _{10}(P)>3.5\right]$ were considered QTLs significantly associated with the traits. The QTL interval was the size of the significant LD block. Each candidate QTL (significant LD block) was checked for known $R$ genes/loci in review papers (Ashkani et al. 2016; Sharma et al. 2012) and recent literature (Kang et al. 2016; Liu et al. 2015; Ma et al. 2014, 2015; Mgonja et al. 2016; Su et al. 2015; Xu et al. 2014; Zhao et al. 2011; Zhu et al. 2016). Previously identified blast QTLs and defenserelated candidate genes were also checked according to the reference Nipponbare genomic sequences (MSU7) in the Gramene database (http://www.gramene.org/), Rice Genome Annotation Project website (http://rice.plant biology.msu.edu/) (Kawahara et al. 2013), and The Rice Annotation Project website (http://rapdb.dna.affrc.go. jp/index.html) (Kawahara et al. 2013; Sakai et al. 2013). Candidate genes were determined by their gene ontology (GO) terms and gene descriptions.

\section{Haplotype analysis}

To identify possible resistance (R) and susceptible (S) haplotypes for the regions significantly associated with resistance, haplotype association analysis was performed with Haploview. Phenotype data were converted to case-control datasets. According to the frequency distributions of LT data (Fig. 1a, c), the accessions showing $\mathrm{LT}>3$ ( $\mathrm{S}$ lesions) and $\leq 3$ ( $\mathrm{R}$ lesions) were assigned as "cases" and "controls", respectively. There is no standard way to determine a threshold for the quantitative DLA data, so DLA $=15 \%$ was arbitrarily set as the threshold for DLA data conversion. For rice accessions inoculated with D41-2, the ratios of "cases (S): controls (R)" for LT and DLA were 168:136 and 210:94, respectively, and with
12YL-DL-3-2, the ratios were 142:86 and 142:86, respectively. In each LD block, the resistance/susceptibility haplotype was determined by Chi-square analysis and $P$ value for haplotype frequencies in cases versus controls. Putative $\mathrm{R}$ and $\mathrm{S}$ haplotypes in all candidate regions were identified by using the SNP genotypes of the $44 \mathrm{~K}$ SNP data. Haplotype identity was assigned if the SNP genotypes were $100 \%$ identical in length and sequences. Pearson correlation analysis was used for analysis of correlation between the resistance performance (LT and DLA) and total number of non-overlapped R/S haplotypes (in each rice accession) by using SAS 6.1 (SAS Inst. Inc., Cary, NC, USA). The total number of nonoverlapped R or S haplotypes was defined as the sum of the number of $\mathrm{R}$ or $\mathrm{S}$ haplotypes identified from different phenotype datasets. The $\mathrm{R}$ or $\mathrm{S}$ haplotype identified from more than one phenotype was counted only once.

\section{Results}

\section{Phenotypic variation of blast resistance in RDP1}

Phenotyping data consistent in all replications were obtained from 304 rice accessions [104 indica (54 indica and 50 aus), 155 japonica (78 TEJ, 72 TRJ, 5 aromatic), and 45 admixed] inoculated with M. oryzae isolate D412, and 228 rice accessions [80 indica (48 indica and 32 aus), 121 japonica (56 TEJ, 63 TRJ, 2 aromatic), and 27 admixed] inoculated with $M$. oryzae isolate 12YL-DL3-2 (Additional file 1: Table S1). Most of the eliminated accessions did not grow well in all the replications. The distributions of LT and DLA are shown for the full population (Fig. 1a-d) and for indica and japonica subgroups (Fig. 1e-h). The distributions of LT were bimodal with discrete resistance $(\mathrm{LT} \leq 3)$ and susceptibility $(\mathrm{LT}>3)$ categories (Fig. 1a, c). LT and DLA were positively correlated (full population with D41-2: $r=0.58, P<0.001$; full population with 12YL: $r=0.66, P<0.001)$. Overall, 55 accessions were resistant (LT in each trial $=0,1,3$ ) and 89 accessions were susceptible (LT in each trial $=5,7,9$ ) to both isolates. The mean of LT and DLA were lower and the resistance was relatively greater for the indica than japonica subgroup (Fig. 1e-h).

\section{Identification of loci associated with resistance to two $M$. oryzae isolates}

Manhattan plots and Q-Q plots for LT and DLA in the full population and indica and japonica subgroups are in Figs. 2, 3. For each experimental dataset, the optimal model for GWAS was selected from GLM, GLM$\mathrm{Q}, \mathrm{MLM}-\mathrm{K}$, and MLM-K+Q. MLM-K+Q had the best explanatory power for global accessions, and GLM and MLM-K were optimal for GWAS at the subgroup level. The strategy of using different optimal models for different traits in the same populations was also adopted by 


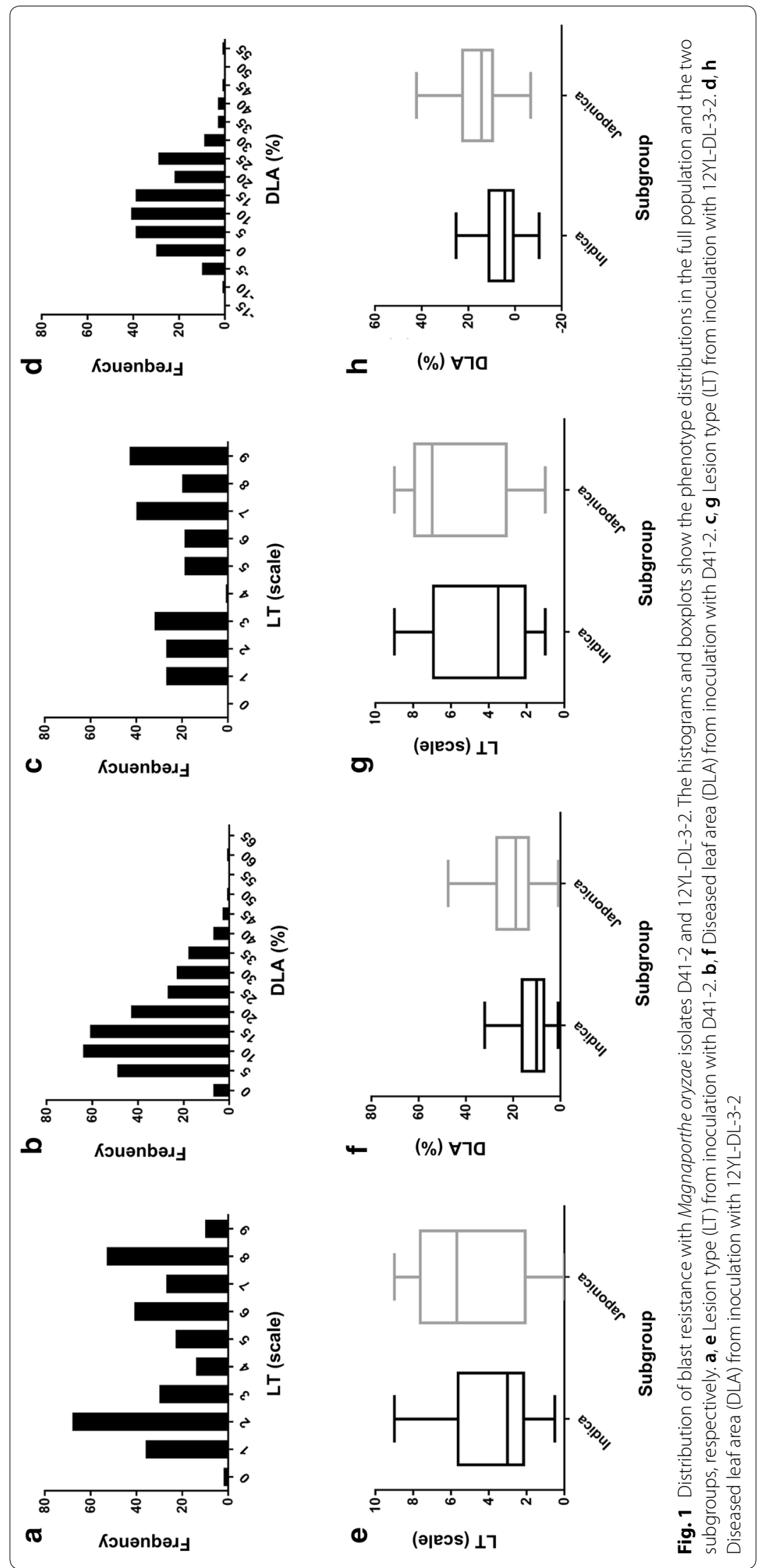


a

Full
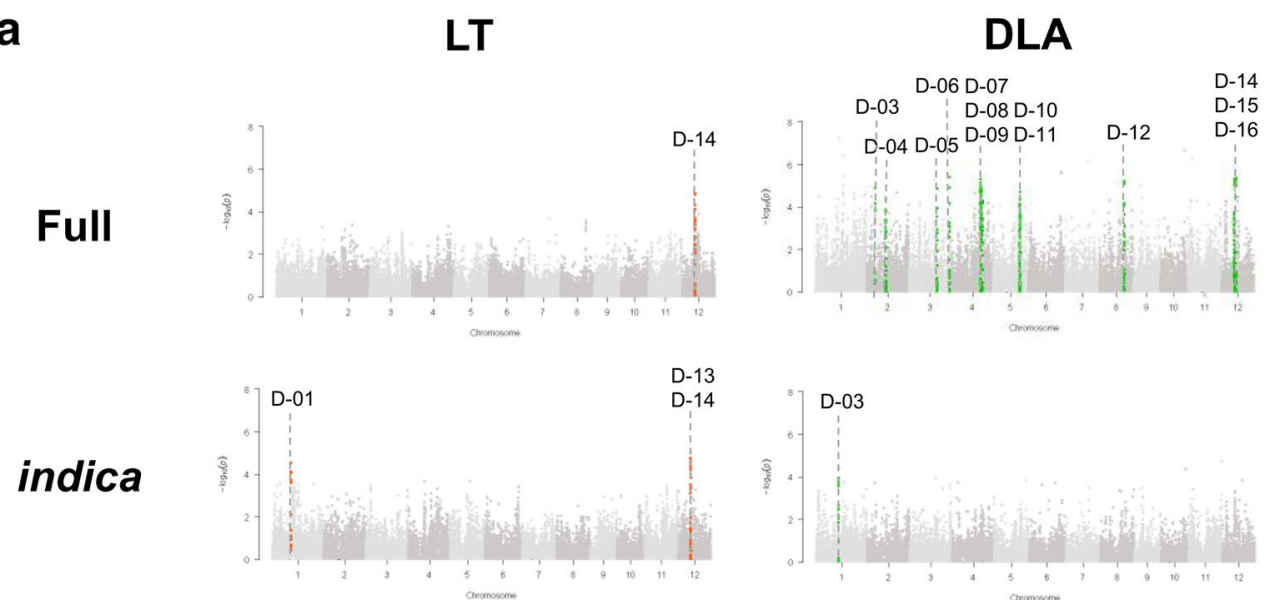

japonica

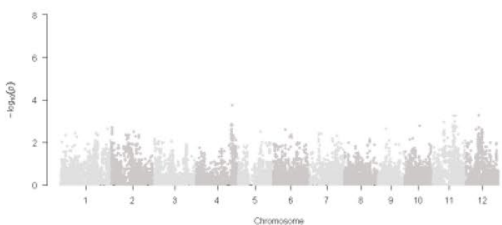

LT

b

Full

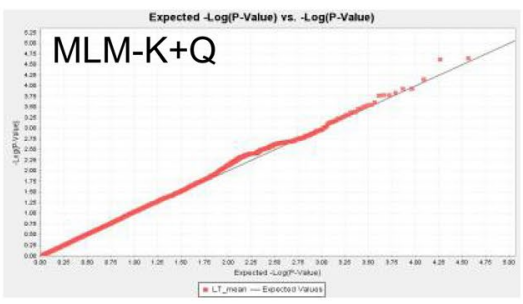

indica
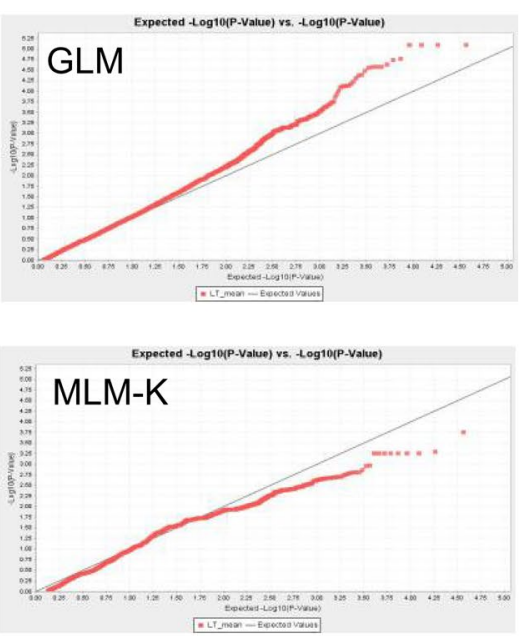
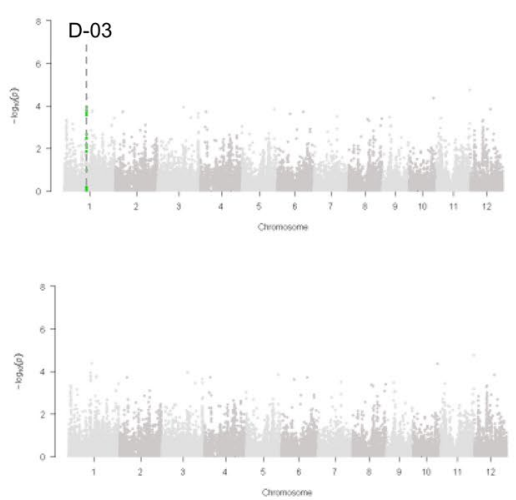

\section{DLA}
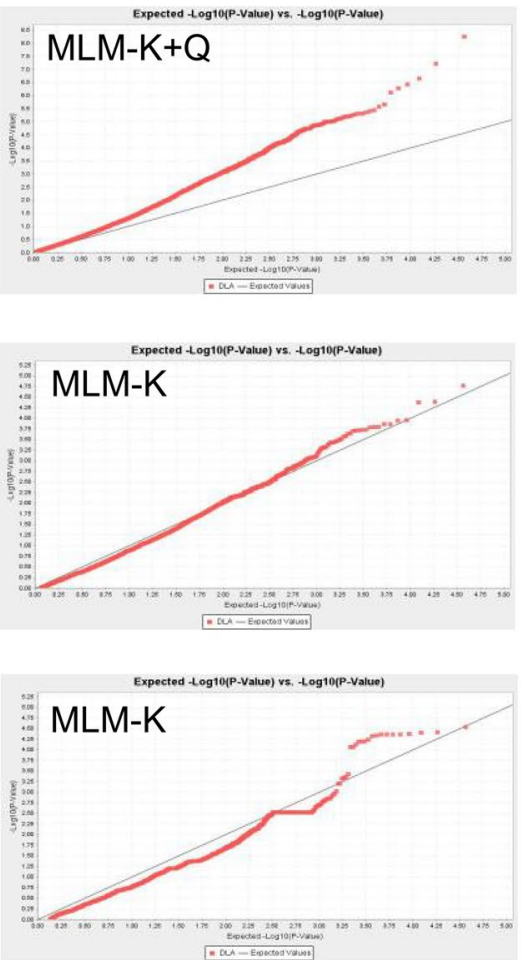

Fig. 2 Genome-wide association study (GWAS) of loci associated with resistance to M. oryzae isolate D41-2. a Manhattan plots show significant genomic regions (D-01 to D-16) identified by using lesion type (LT) and diseased leaf area (DLA) dataset. $X$ axis: rice chromosomes; $Y$ axis: - $\log _{10}(P)$. b Quantile-quantile (Q-Q) plots show the fitness of the selected models used for different traits in the full population or subgroups. $X$ axis: expected $-\log _{10}(P) ; Y$ axis: observed $-\log _{10}(P)$ 
a

Full
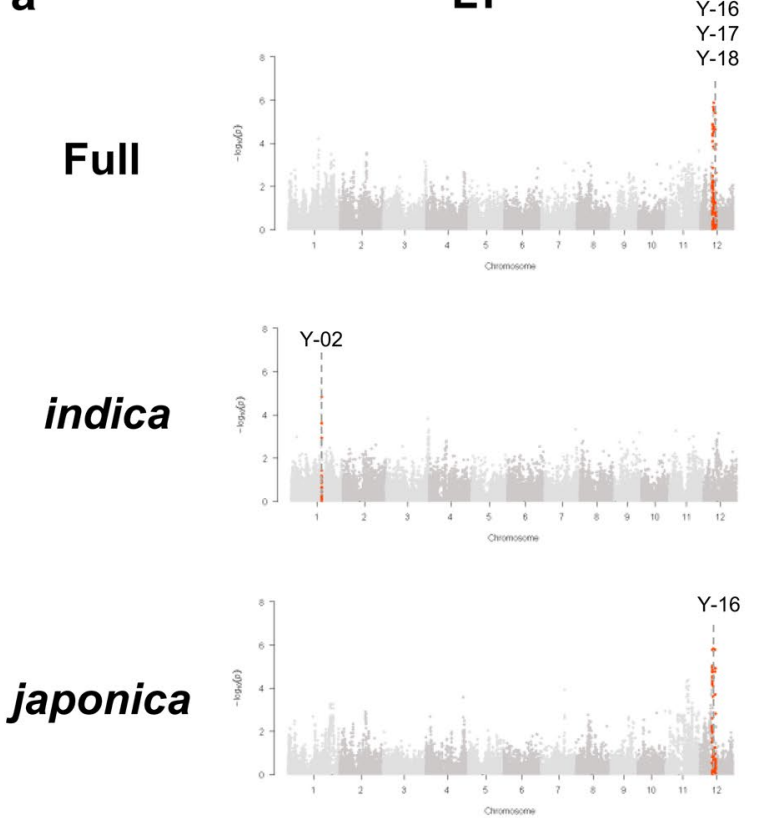

b

Full

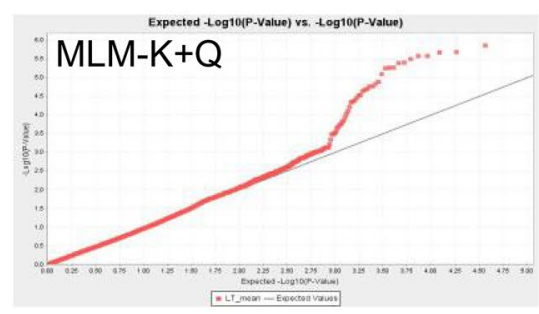

LT
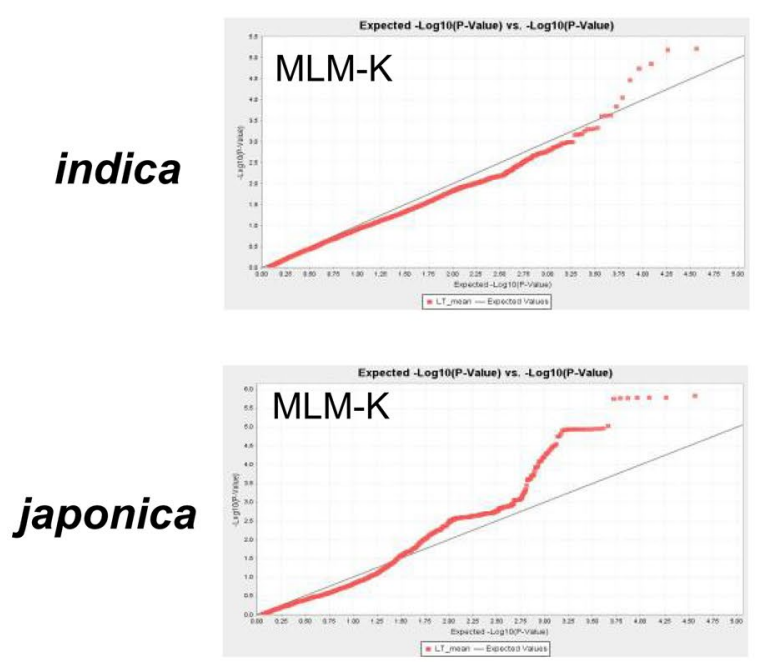

DLA
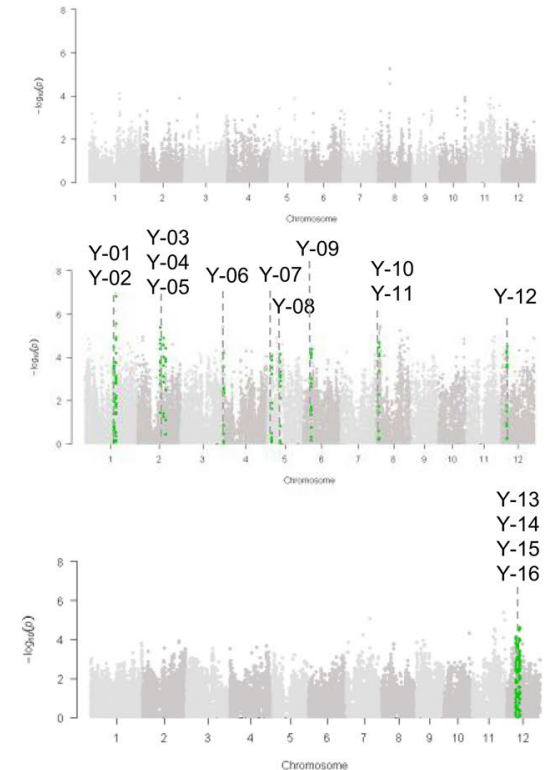

DLA
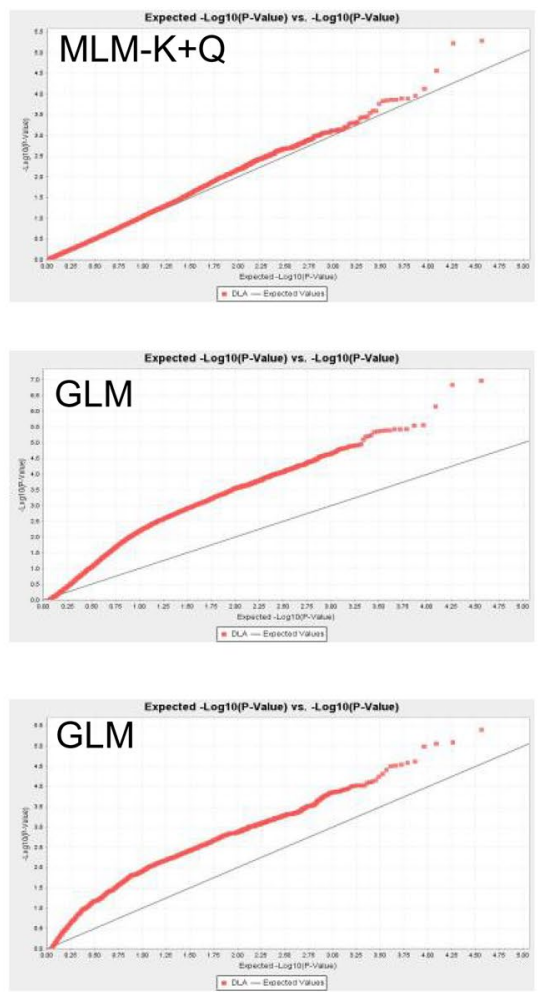

Fig. 3 GWAS of loci associated with resistance to M. oryzae isolate 12YL-DL-3-2. a Manhattan plots show significant genomic regions (Y-01 to Y-18) identified by using lesion type (LT) and diseased leaf area (DLA) datasets. X axis: rice chromosomes; $Y$ axis: $-\log _{10}(P)$. b Quantile-quantile $(\mathrm{Q}-\mathrm{Q})$ plots show the fitness of the selected models used for different traits in the full population or subgroups. $X$ axis: $\operatorname{expected}_{-} \log _{10}(P) ; Y$ axis: observed $-\log _{10}(P)$ 
Gajardo et al. (2015), Jaiswal et al. (2016), and Wan et al. (2017).

A total of 32 non-overlapped loci associated with blast resistance were identified across 8 of the 12 rice chromosomes (no QTL identified in chromosomes 7, 9, 10 and 11). The number of QTLs identified from different phenotypes, populations, and M. oryzae isolates are summarized in Additional file 3: Table S3. Overall, 16 and 18 QTLs were detected with D41-2 (D-01 to D-16; Table 1 and Fig. 2) and 12YL-DL3-2 (Y-01 to Y-18; Table 2 and Fig. 3), respectively. Four QTLs (D-13/Y-16, D-14, D-15/Y-17, and Y-02) were identified for both DLA and LT, and more QTLs were mapped from the indica than japonica subpopulation. Two QTLs were identified with both isolates: D-13 and Y-16 overlapped at 10.21-10.61 Mb, and D-15 and Y-17 were both located at $11.06-11.56 \mathrm{Mb}$ in chromosome 12 . Detailed information on the QTL intervals, the most significantly associated markers, and the co-localized $R$ genes/QTLs are in Tables 1 and 2. In total, 6 and 30 QTLs were detected for LT and DLA, respectively, with $-\log _{10}(P)$ values ranging from 3.72 to 6.83 and the proportion of phenotypic variation explained $\left(R^{2}\right)$ from 0.02 to 0.31 . GWAS of global accessions and within varietal subgroups revealed different QTLs. In all, 13 of the 16 QTLs against D41-2 were detected in the full population, and 12 of the 18 QTLs against 12YL-DL3-2 were detected specifically in the indica subgroup. D-13/Y-16 and D-14 were the common QTLs identified at both the full-population and subgroup levels.

Among the 32 identified candidate QTLs, 22 regions co-localized with previously reported $R$ genes and/or QTLs, including two cloned $R$ genes, Pita (D-13/Y-16) and Ptr (D-14); 19 mapped $R$ loci and 20 mapped QTLs. Only 3 regions co-localized with the QTLs identified in other GWAS of RDP1: D-13/Y-16 co-localized with Pita (Zhao et al. 2011), LABR_87, LABR_88, and LABR_89 (Kang et al. 2016); D-15/Y-17 co-localized with LABR_90 (Kang et al. 2016); and Y-01 co-localized with LABR_10 (Kang et al. 2016). For some reported large-interval $R$ loci and QTLs, more than one candidate QTL was detected within the ranges. On chromosome 1, D-01 and D-02 were detected within AQCT001, AQEN001, AQEN011, and AQEN018, and Y-01 and Y-02 were detected within AQCT001 and AQEN001. On chromosome 4, D-07 to D-09 were detected within CQAC2; on chromosome $12, \mathrm{D}-13$ to D-15 and Y-14 to Y-18 were detected within AQCT008, AQEN010, AQEN017, Pi6 $(t)$, Pi12, Pi19(t), Pi62 $(t), P i 157$, and Pita2. In addition to known $R$ genes or QTLs, we detected 10 new genomic regions associated with blast resistance on chromosome 2 (D-03, D-04, Y-03, Y-05), chromosome 3 (D-06, Y-06), chromosome 5 (D-11, Y-07), and chromosome 8 (Y-10, Y-11).
A total of 100 defense-related genes were identified within the intervals of the 32 QTLs (Additional file 4: Table S4). These included NBS-LRR genes, receptor-like protein kinase (RLK) genes, transcription factor genes, ubiquitination-related genes, and oxidase/oxidoreductase genes.

\section{Resistance and susceptibility haplotypes in RDP1}

LD analyses defined a total of 3261 (full population), 3226 (indica subgroup) and 1644 (japonica subgroup) LD blocks in the rice genome. Each block contained 3-13 haplotypes. Putative R haplotypes (frequency of control $>$ frequency of case) and S haplotypes (frequency of case $>$ frequency of control) of the 32 QTL regions in all the tested rice accessions are shown in Additional file 5: Table S5 and Additional file 6: Table S6. For each accession, $\mathrm{R}$ haplotypes were observed from 0 to $10 \mathrm{QTL}$ regions and $\mathrm{S}$ haplotypes were observed from 0 to 11 QTL regions. For both isolates, the total number of nonoverlapped $\mathrm{R}$ haplotypes was negatively correlated with disease severity $(r=-0.29$ to $-0.41, P<0.001)$, but the total number of non-overlapped $\mathrm{S}$ haplotypes was positively correlated with disease severity ( $r=0.28$ to 0.41 , $P<0.001$ ) (Additional file 7: Table S7).

To apply the haplotype information in Additional file 5: Table S5 and Additional file 6: Table S6 for resistance breeding, one can first choose resistance donors based on the resistance performance data in rows 3-6 (DLA and LT from D41-2 and 12YL-DL3-2 inoculations), then scroll down to find the QTLs likely contributing resistance in each accession. For example, NSFTV 17 exhibited good resistance to both $M$. oryzae isolates (DLA $=8.95$ and 2.58; $\mathrm{LT}=1.7$ and 3$)$, and it may contain resistant QTLs D-08, D-09, and D-14 against D41-2 (Additional file 5: Table S5) and resistant QTLs Y-02, Y-03, Y-07, Y-08, Y-09, and Y-11 against 12YL-DL3-2 (Additional file 6: Table S6). If a certain QTL is of interest, one can easily find accessions containing putative $\mathrm{R}$ or $\mathrm{S}$ haplotypes in this region. For instance, NSFTV 6 and NSFTV 10 may be used as resistance and susceptible donors for D-05, respectively (Additional file 5: Table S5). With the open access $44 \mathrm{~K}$ or $700 \mathrm{~K}$ genotypic data, molecular markers targeting candidate QTL can be designed and applied in marker-assisted selection programs.

\section{Discussion}

GWAS has been widely used for high-resolution mapping and mining for resistance resources in plants. In this research, we identified 32 non-overlapped regions associated with resistance to blast infection in rice by inoculating the open-access RDP1 with two representative $M$. oryzae isolates from Taiwan. Most of the QTLs were identified by inoculation of D41-2 or 12YL-DL3-2, 


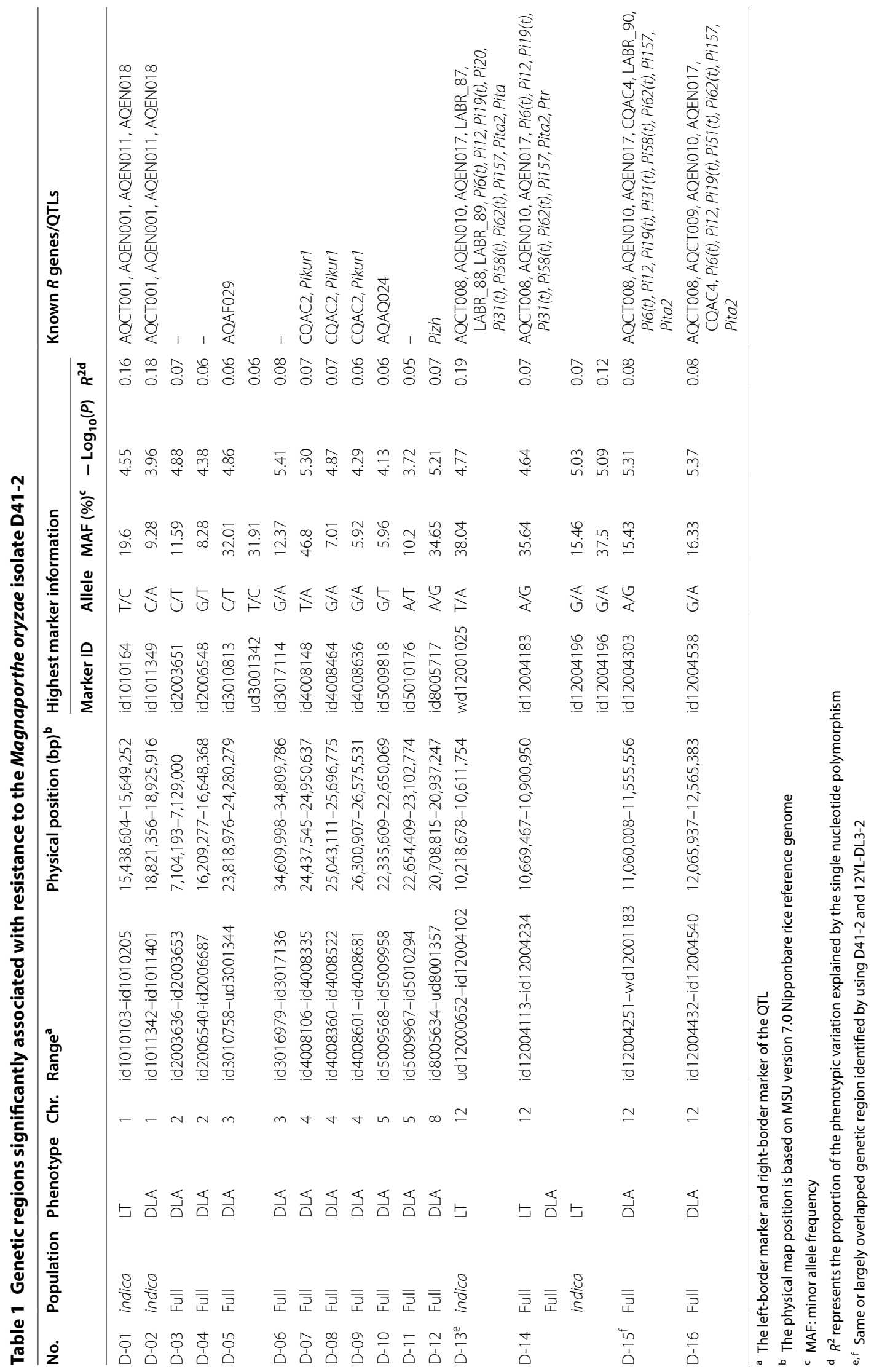




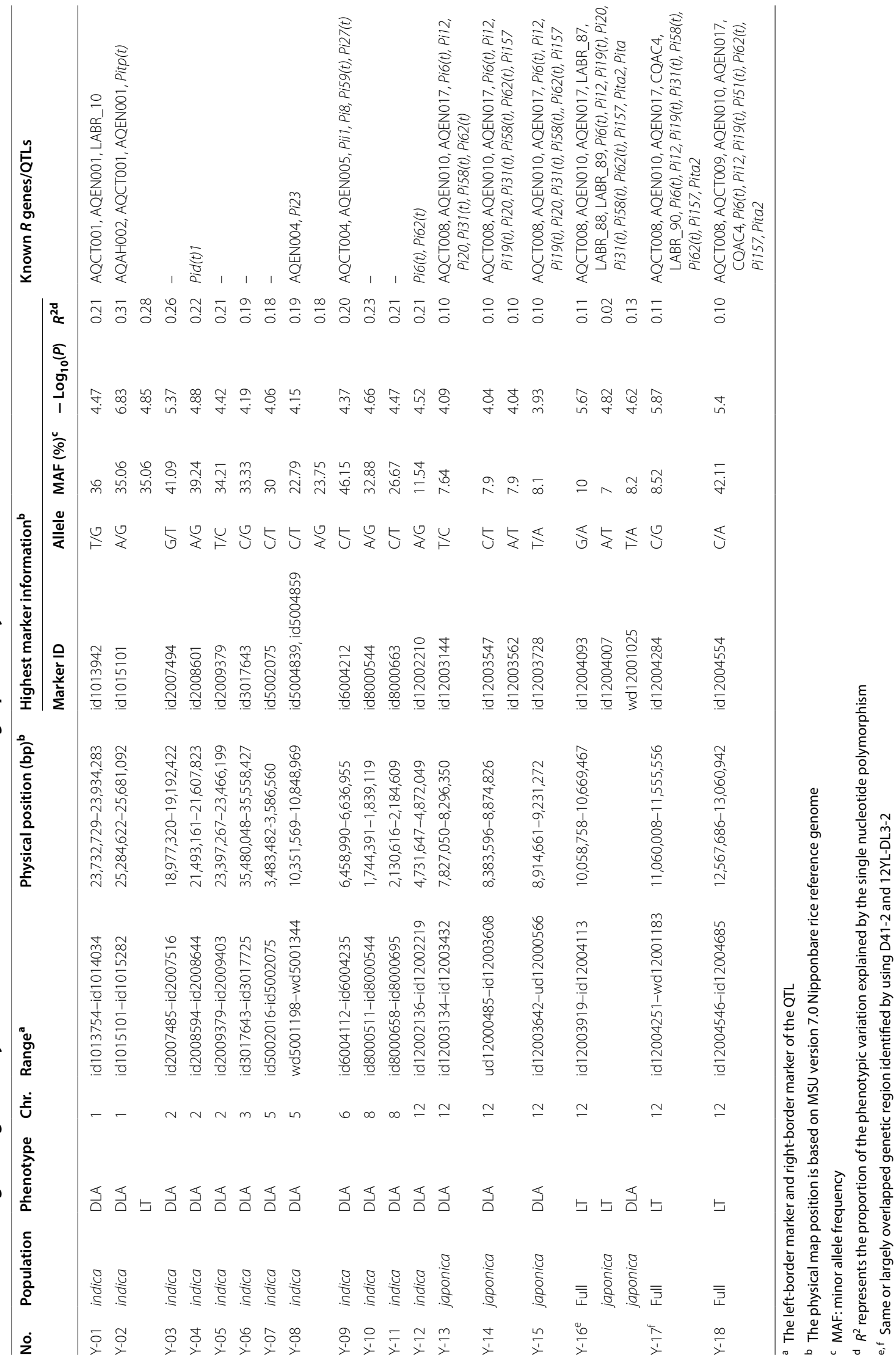


but two QTLs, D-13/Y-16 and D-15/Y-17, on chromosome 12, were resistant to both isolates. The blast resistance QTLs mapped in our and other studies using RDP1 largely differ (Kang et al. 2016; Mgonja et al. 2016; Zhao et al. 2011; Zhu et al. 2016). In agreement with Kang et al. (2016), we identified some QTLs specifically in the indica or japonica subgroup, which may be associated with the frequencies of different resistant alleles in the two subgroups.

Among the QTLs detected in the present study, D-13/Y-16 on chromosome 12 showed the strongest resistance to both isolates. D-13/Y-16 had the greatest number of significant SNPs, high $-\log _{10}$ (P-value), and high $R^{2}$ value (Tables 1 and 2). The QTL is located at $10.05-10.67 \mathrm{Mb}$ on chromosome 12 , a region containing Pita (10.60-10.62 Mb), and co-localized with numerous $R$ loci [Pi6(t), Pi12, Pi19(t), Pi20, Pi31(t), Pi58(t), Pi62(t), Pi157, Pita2] and reported QTLs. The neighboring D-14 $(10.67-10.90 \mathrm{Mb})$ contains Ptr (LOC_Os12g18729; $10.82-10.84 \mathrm{Mb}$ ), an atypical $R$ gene required for Pitaand Pita-2-mediated resistance (Zhao et al. 2018). The Pita locus was also identified as a major QTL in RDP1 by Kang et al. (2016) and Zhao et al. (2011). D41-2 and 12YLDL3-2 are compatible with IRBLTA-K1, IRBLTA-CT2, and IRBLTA-CP1, the monogenic lines carrying Pita in the LTH background (unpublished data), so the major QTL we detected is likely allelic or linked to Pita. In addition to Pita (LOC_Os12g18360), LOC_Os12g17550 (10.05-10.07 Mb), encoding an LRR-containing protein, and LOC_Os12g18374 (10.62-10.64 Mb), encoding an NB-ARC-containing protein, are potential causal genes. As well, D-13 was identified from the indica subgroup, and Y-16 was identified from the full population and the japonica subgroup, which suggests the presence of diverse resistant variants of Pita and/or other linked $R$ genes in indica and japonica rice accessions.

Previous GWAS of blast resistance relied on the 0-9 scoring systems that combine the evaluation of lesion size, LT and DLA by the naked eye (IRRI 2014; Kang et al. 2016; Mgonja et al. 2016; Zhao et al. 2011). In this study, we photographed the diseased samples, and LT and DLA were independently assessed by using image analysis. The use of LT for GWAS revealed only 6 QTLs on chromosome 1 (D-01: 15.43-15.65 Mb; Y-02: 25.28-25.68 Mb) and chromosome 12 (D-13/Y-16: $10.05-10.67 \mathrm{Mb}$; D-14: 10.66-10.90 Mb; Y-17: 11.06-11.56 Mb; Y-18: 12.56-13.06 Mb). An additional 26 QTLs were identified when the DLA trait was analyzed. Hence, the detailed phenotype data and accurate quantification of DLA can improve the power of QTL detection.

Our association mapping results can serve as a good reference for finer delimitation of previously reported large-interval $R$ loci and QTLs. Considering that significant SNP signals may not locate nearby or within the coding regions of causative genes (Kang et al. 2016), we present the candidate QTLs from this study based on LD blocks. The mean interval of our candidate QTLs was $309 \mathrm{~kb}$ (range 25 to $654 \mathrm{~kb}$ ), which is higher than the resolution from conventional linkage mapping studies. In fact, many previously identified blast $R$ loci were located at $>1000 \mathrm{~kb}$ genetic regions (Sharma et al. 2012).

A variety of candidate genes were identified within the QTL regions. According to the annotation, these genes may be involved in recognition, signaling, and/or antimicrobial activities in effector-triggered immunity (ETI) or pathogen-associated molecular pattern (PAMP)-triggered immunity (PTI) in plants (Jones and Dangl 2006). For instance, in D-05, the $-\log _{10}(P)$ peak and four significant SNPs were detected within a gene encoding an LRR protein (LOC_Os03g43390; 24.19-24.20 Mb on chromosome 3) (Additional file 8: Fig. S1A). This $R$-like gene is worthy of further investigation. A cluster of 21 significant SNPs with strong LD (LD parameter $r^{2}>0.8$ ) was found across D-08 (Additional file 8: Fig. S1B), which co-localizes with a large-interval $R$ locus Pikur1 on chromosome 4 (Goto 1970). Genes encoding LRR proteins (LOC_Os04g42470, LOC_Os04g42670, LOC_Os04g43340) and ubiquitin-related proteins were found in the D-08 interval. Ubiquitination is known to be involved in the modulation of (compatible and incompatible) plant-pathogen interactions via posttranslational modifications of the protein components of PTI and ETI (Park et al. 2012; Shirsekar et al. 2010). Fine-mapping and functional assays will be needed to validate the causal genes underlying the identified QTLs.

A number of rice accessions in RDP1 have great potential for resistance breeding and further exploitation. LAC 23 (NSFTV 99) and OS 6 (WC 10296, NSFTV 395) showed resistance to both Taiwanese isolates (LT $<3$ and DLA $<10 \%$ ); they were also reported to be resistant against isolates from China, South Korea, Columbia, Philippines, India, and the United States (Kang et al. 2016; Zhao et al. 2011; Zhu et al. 2016). The mechanisms of broad-spectrum resistance are worthy of further exploration. Our haplotype analysis revealed that in general, the more $\mathrm{R}$ haplotypes detected in an accession, the greater the degree of resistance. Eight accessions (NSFTV 17, NSFTV 135, NSFTV 161, NSFTV 183, NSFTV 202, NSFTV 235, NSFTV 642) carried $\geq 7 \mathrm{R}$ haplotypes and were resistant to both isolates. These accessions are particularly useful for cultivar improvement in Taiwan. Moreover, Pi27(t) was originally identified from the resistance donor IR64 (NSFTV 644). This accession was included in RDP1 and the resistance haplotype was accurately assigned to 
the QTL region (Y-09) corresponding to Pi27(t), which suggests the validity of the haplotype identification analysis in this study.

\section{Conclusions}

This study used the GWAS approach to explore blast resistance in the open-access RDP1. Although RDP1 had been used to locate blast resistance QTLs in a few recent studies (Zhao et al. 2011; Kang et al. 2016; Mgonja et al. 2016; Zhu et al. 2016), the QTLs mapped with geographically distinct $M$. oryzae isolates were largely different from those in our study. This observation indicates the diverse composition of $A v r$ genes in geographically distinct $M$. oryzae isolates and the richness of qualitative and quantitative resistance genes in RDP1. By conventional visual rating and the use of image analysis for accurate quantitative assessment of disease severity, we delineated 32 known and new genomic regions controlling blast resistance and identified 100 candidate genes encoding leucine-rich repeat-containing proteins, transcription factors, ubiquitination-related proteins, and peroxidases. We also determined putative resistance and susceptibility haplotypes of the 32 QTLs for each tested rice accession. The information provided in Additional file 5: Table S5 and Additional file 6: Table S6 will aid in selecting suitable resistance donor lines for gene/QTL validation and further application.

\section{Additional files}

Additional file 1: Table S1. Rice accessions evaluated with Magnaporthe oryzae isolates D41-2 and 12YL-DL-3-2.

Additional file 2: Table S2. Reaction patterns of international standard blast differential varieties (ID1 to ID8) and Taiwan blast differential varieties (TD1 to TD16) to M. oryzae isolates D41-2 and 12YL-DL3-2.

Additional file 3: Table S3. Number of QTLs identified from different phenotypes, populations, and M. oryzae isolates.

Additional file 4: Table S4. Candidate genes identified within the regions associated with blast resistance.

Additional file 5: Table S5. Resistance and susceptibility haplotypes in the candidate blast QTLs identified using Magnaporthe oryzae isolate D41-2.

Additional file 6: Table S6. Resistance and susceptibility haplotypes in the candidate blast QTLs identified using Magnaporthe oryzae isolate 12YL-DL3-2

Additional file 7: Table S7. Pearson correlation coefficient ( $r$ ) for the association between the level of disease severity and the total number of non-redundant resistance (R) or susceptible (S) haplotypes in tested accessions.

Additional file 8: Fig. S1. Linkage disequilibrium (LD) of the significant single nucleotide polymorphisms (SNPs) in the candidate regions D-05 and D-08. The values of LD parameter $\left(r^{2}\right)$ were calculated between each SNP and the SNP with the highest $-\log _{10}(P)$.

\section{Abbreviations}

$\% D L A$ : proportion of diseased leaf area; BLUEs: best linear unbiased estimates; ETI: effector-triggered immunity; GLM: generalized linear model; GO: gene ontology; GSOR: Genetics Stocks Oryza; GWAS: genome-wide association study; LD: linkage disequilibrium; LT: lesion type; MLM: mixed linear model; NBS-LRR: nucleotide binding site-leucine-rich repeat; OMA: oat meal agar; PAMP: pathogen-associated molecular pattern; PTI: pathogen-associated molecular pattern-triggered immunity; Q-Q: quantile-quantile; QTL: quantitative trait loci; RCBD: randomized complete block design; RDP1: rice diversity panel 1; R genes: resistance genes; RLK: receptor-like protein kinase; SNP: single-nucleotide polymorphism; TEJ: temperate japonica; TRJ: tropical japonica.

\section{Authors' contributions}

HAL, SYC, FYC, and CLC conceived and designed the experiments. HAL performed the experiments. CWT helped in GWAS. YCC performed the haplotype analysis. WCS and RSC provided M. oryzae isolates and helped in fungal culture. FYC and CWW amplified the seeds of RDP1. HAL, SYC, and CLC analyzed the data and wrote the paper. All authors read and approved the final manuscript.

\section{Author details}

${ }^{1}$ Department of Plant Pathology and Microbiology, National Taiwan University, No. 1, Sec. 4, Roosevelt Rd., Taipei 10617, Taiwan. ${ }^{2}$ Kaohsiung District Agricultural Research and Extension Station, No. 2-6, Dehe Rd., Pingtung County 90846, Taiwan. ${ }^{3}$ Department of Agronomy, National Taiwan University, No. 1, Sec. 4, Roosevelt Rd., Taipei 10617, Taiwan. ${ }^{4}$ Department of Biochemical Science and Technology, National Chiayi University, No. 300, Syuefu Rd., Chiayi City 60004, Taiwan.

\section{Acknowledgements}

We thank Khong-Sam Chia, Yi-Jui Lo, and Hao Li for help with seed amplification; Jui-Yu Liao, Yu-Chia Chen, Wei-Lun Chen, An-Po Cheng, Yu-Cyuan Shih, Qiao-Juan Lai, and Shun-Yuan Huang for participating in phenotyping; and Wei-Bin Chang and Hsin-Han Lee for assisting data analysis.

\section{Competing interests}

The authors declare that they have no competing interests.

Availability of data and materials

Not applicable.

Consent for publication

Authors agree to the terms of the Springer Open Copyright and License Agreement.

\section{Ethics approval and consent to participate}

Not applicable.

\section{Funding}

The work was supported by the Ministry of Science and Technology of Taiwan (102-2313-B-002-065-MY3; 105-2313-B-002-014-; 106-2313-B-002-021-MY3) and Bureau of Animal and Plant Inspection and Quarantine (BAPHIQ), Council of Agriculture, Taiwan [104AS-10.6.1-BQ-B1(1); 105AS-10.5.4-BQ-B1(1); 106AS-9.5.4-BQ-B1(1); 107AS-8.4.4-BQ-B1(1)].

\section{Publisher's Note}

Springer Nature remains neutral with regard to jurisdictional claims in published maps and institutional affiliations.

Received: 19 July 2018 Accepted: 12 December 2018

Published online: 21 December 2018

\section{References}

Ashkani S, Rafii MY, Shabanimofrad M, Ghasemzadeh A, Ravanfar SA, Latif MA (2016) Molecular progress on the mapping and cloning of functional genes for blast disease in rice (Oryza sativa L.): current status and future considerations. Crit Rev Biotechnol 36:353-367 
Azizi P, Rafii MY, Mahmood M, Abdullah SN, Hanafi MM, Nejat N, Latif MA, Sahebi M (2015) Differential gene expression reflects morphological characteristics and physiological processes in rice immunity against blast pathogen Magnaporthe oryzae. PLoS ONE 10(5):e0126188

Barrett JC, Fry B, Maller J, Daly MJ (2005) Haploview: analysis and visualization of LD and haplotype maps. Bioinformatics 21:263-265

Begum H, Spindel JE, Lalusin A, Borromeo T, Gregorio G, Hernandez J, Virk P, Collard B, McCouch SR (2015) Genome-wide association mapping for yield and other agronomic traits in an elite breeding population of tropical rice (Oryza sativa). PLOS ONE 10:e0119873

Bradbury PJ, Zhang Z, Kroon DE, Casstevens TM, Ramdoss Y, Buckler ES (2007) TASSEL: software for association mapping of complex traits in diverse samples. Bioinformatics 23:2633-2635

Buckler ES, Holland JB, Bradbury PJ, Acharya CB, Brown PJ, Browne C, Ersoz E, Flint-Garcia S, Garcia A, Glaubitz JC, Goodman MM, Harjes C, Guill K, Kroon DE, Larsson S, Lepak NK, Li H, Mitchell SE, Pressoir G, Peiffer JA, Rosas MO, Rocheford TR, Romay MC, Romero S, Salvo S, Sanchez Villeda H, da Silva HS, Sun Q, Tian F, Upadyayula N, Ware D, Yates H, Yu J, Zhang Z, Kresovich S, McMullen MD (2009) The genetic architecture of maize flowering time. Science 325:714-718

Chen X, Shang J, Chen D, Lei C, Zou Y, Zhai W, Liu G, Xu J, Ling Z, Cao G, Ma B, Wang Y, Zhao X, Li S, Zhu L (2006) A B-lectin receptor kinase gene conferring rice blast resistance. Plant J 46:794-804

Chen LC, Huang SH, Cheng CH (2009) Review of the screening tests for rice varietal resistance to major diseases and insect pests in Taiwan. In: Ni HF, Yang HR (eds) Proceedings of symposium on achievements and perspectives of rice protection in Taiwan, 161p, 1st ed. Chiayi Agricultural Experiment Branch, Taiwan Agricultural Research Institute, Chiayi, Taiwan. pp 83-103

Collard BCY, Jahufer MZZ, Brouwer JB, Pang ECK (2005) An introduction to markers, quantitative trait loci (QTL) mapping and marker-assisted selection for crop improvement: the basic concepts. Euphytica 142:169-196

Das A, Soubam D, Singh PK, Thakur S, Singh NK, Sharma TR (2012) A novel blast resistance gene, Pi54rh cloned from wild species of rice, Oryza rhizomatis confers broad spectrum resistance to Magnaporthe oryzae. Funct Integr Genomics 12:215-228

Deng Y, Zhu X, Xu J, Chen H, He Z (2009) Map-based cloning and breeding application of a broad-spectrum resistance gene Pigm to rice blast. Advances in genetics, genomics and control of rice blast disease. Springer, Dordrecht, pp 161-171

Eizenga GC, Ali M, Bryant RJ, Yeater KM, McClung AM, McCouch SR (2014) Registration of the rice diversity panel 1 for genomewide association studies. J Plant Regist 8:109-116

Famoso AN, Zhao K, Clark RT, Tung C-W, Wright MH, Bustamante C, Kochian LV, McCouch SR (2011) Genetic architecture of aluminum tolerance in rice (Oryza sativa) determined through genome-wide association analysis and QTL mapping. PLoS Genet 7:e1002221

Food and Agriculture Organization of the United Nations (2016) FAO Rice Market Monitor 19:1-31. http://www.fao.org/economic/RMM/en/. Accessed 5 Feb 2017

Fukuoka S, Saka N, Koga H, Ono K, Shimizu T, Ebana K, Hayashi N, Takahashi A, Hirochika H, Okuno K, Yano M (2009) Loss of function of a prolinecontaining protein confers durable disease resistance in rice. Science 325:998-1001

Fukuoka S, Yamamoto SI, Mizobuchi R, Yamanouchi U, Ono K, Kitazawa N, Yasuda N, Fujita Y, Thanh Nguyen TT, Koizumi S, Sugimoto K, Matsumoto T, Yano M (2014) Multiple functional polymorphisms in a single disease resistance gene in rice enhance durable resistance to blast. Sci Rep 4:4550

Gajardo HA, Wittkop B, Soto-Cerda B, Higgins EE, Parkin IA, Snowdon RJ, Federico ML, Iniguez-Luy FL (2015) Association mapping of seed quality traits in Brassica napus L. using GWAS and candidate QTL approaches. Mol Breed 35:143

Goto I (1970) Genetic studies on the resistance of rice plant to the blast fungus. I. Inheritance of resistance in crosses Sensho $\times$ H-79 and Imochishirazu x H-79. Ann Phytopathol Soc Jpn 36:304-312

Huang X, Han B (2014) Natural variations and genome-wide association studies in crop plants. Annu Rev Plant Biol 65:531-551

Huang X, Wei X, Sang T, Zhao Q, Feng Q, Zhao Y, Li C, Zhu C, Lu T, Zhang Z, Li M, Fan D, Guo Y, Wang A, Wang L, Deng L, Li W, Lu Y, Weng Q, Liu K, Huang T, Zhou T, Jing Y, Li W, Lin Z, Buckler ES, Qian Q, Zhang Q-F, Li J, Han B
(2010) Genome-wide association studies of 14 agronomic traits in rice landraces. Nat Genet 42:961-967

Huang X, Zhao Y, Wei X, Li C, Wang A, Zhao Q, Li W, Guo Y, Deng L, Zhu C, Fan D, Lu Y, Weng Q, Liu K, Zhou T, Jing Y, Si L, Dong G, Huang T, Lu T, Feng Q, Qian Q, Li J, Han B (2012) Genome-wide association study of flowering time and grain yield traits in a worldwide collection of rice germplasm. Nat Genet 44:32-39

IRRI (2014) Standard evaluation system for rice, 5th edn. International Rice Research Institute, Manila, p 57

Jaiswal V, Gahlaut V, Meher PK, Mir RR, Jaiswal JP, Rao AR, Balyan HS, Gupta PK (2016) Genome wide single locus single trait, multi-locus and multi-trait association mapping for some important agronomic traits in common wheat (T. aestivum L.). PLoS ONE 11:e0159343

Jones JDG, Dangl JL (2006) The plant immune system. Nature 444:323-329

Kang H, Wang Y, Peng S, Zhang Y, Xiao Y, Wang D, Qu S, Li Z, Yan S, Wang Z, Liu W, Ning Y, Korniliev P, Leung H, Mezey J, McCouch SR, Wang GL (2016) Dissection of the genetic architecture of rice resistance to the blast fungus Magnaporthe oryzae. Mol Plant Pathol 17:959-972

Kawahara Y, de la Bastide M, Hamilton JP, Kanamori H, McCombie WR, Ouyang S, Schwartz DC, Tanaka T, Wu J, Zhou S, Childs KL, Davidson RM, Lin H, Quesada-Ocampo L, Vaillancourt B, Sakai H, Lee SS, Kim J, Numa H, Itoh T, Buell CR, Matsumoto T (2013) Improvement of the Oryza sativa Nipponbare reference genome using next generation sequence and optical map data. Rice 6:4

Koide Y, Kobayashi N, Xu D, Fukuta Y (2009) Resistance genes and selection DNA markers for blast disease in rice (Oryza sativa L.). Jpn Agric Res Q 43:255-280

Kovach MJ, Sweeney MT, McCouch SR (2007) New insights into the history of rice domestication. Trends Genet 23:578-587

Lamari L (2008) Assess 2.0: image analysis software for plant disease quantification. American Phytopathological Society, St. Paul, p 125

Liu Y, Qi X, Young ND, Olsen KM, Caicedo AL, Jia Y (2015) Characterization of resistance genes to rice blast fungus Magnaporthe oryzae in a "Green Revolution" rice variety. Mol Breed 35:52

Ma J, Jia MH, Jia Y (2014) Characterization of rice blast resistance gene Pi61(t) in rice germplasm. Plant Dis 98:1200-1204

Ma J, Lei C, Xu X, Hao K, Wang J, Cheng Z, Ma X, Ma J, Zhou K, Zhang X, Guo X, Wu F, Lin Q, Wang C, Zhai H, Wang H, Wan J (2015) Pi64, encoding a novel CC-NBS-LRR protein, confers resistance to leaf and neck blast in rice. Mol Plant Microbe Interact 28:558-568

Marone D, Russo MA, Laidò G, De Leonardis AM, Mastrangelo AM (2013) Plant nucleotide binding site-leucine-rich repeat (NBS-LRR) genes: active guardians in host defense responses. Int J Mol Sci 14:7302-7326

McCouch SR, Wright MH, Tung C-W, Maron LG, McNally KL, Fitzgerald M, Singh N, DeClerck G, Agosto-Perez F, Korniliev P, Greenberg AJ, Naredo ME, Mercado SM, Harrington SE, Shi Y, Branchini DA, Kuser-Falcão PR, Leung H, Ebana K, Yano M, Eizenga G, McClung A, Mezey J (2016) Open access resources for genome-wide association mapping in rice. Nat Commun 7:10532

McDowell JM, Woffenden BJ (2003) Plant disease resistance genes: recent insights and potential applications. Trends Biotechnol 21:178-183

Mgonja EM, Balimponya EG, Kang H, Bellizzi M, Park CH, Li Y, Mabagala R, Sneller C, Correll J, Opiyo S, Talbot NJ, Mitchell T, Wang GL (2016) Genome-wide association mapping of rice resistance genes against Magnaporthe oryzae isolates from four African countries. Phytopathology 106:1359-1365

Miah G, Rafii MY, Ismail MR, Puteh AB, Rahim HA, Asfaliza R, Latif MA (2013) Blast resistance in rice: a review of conventional breeding to molecular approaches. Mol Biol Rep 40:2369-2388

Norton GJ, Douglas A, Lahner B, Yakubova E, Guerinot ML, Pinson SR, Tarpley L, Eizenga GC, McGrath SP, Zhao F-J, Islam MR, Islam S, Duan G, Zhu Y, Salt DE, Meharg AA, Price AH (2014) Genome wide association mapping of grain arsenic, copper, molybdenum and zinc in rice (Oryza sativa L.) grown at four international field sites. PLoS ONE 9:e89685

Oraguzie NC, Gardiner SE, Rikkerink EH, Silva HN (2007) Association mapping in plants. Springer, New York, p 277

Park CH, Chen S, Shirsekar G, Zhou B, Khang CH, Songkumarn P, Afzal AJ, Ning Y, Wang R, Bellizzi M, Valent B, Wang GL (2012) The Magnaporthe oryzae effector AvrPiz-t targets the RING E3 ubiquitin ligase APIP6 to suppress pathogen-associated molecular pattern-triggered immunity in rice. Plant Cell 24:4748-4762 
Pasam RK, Sharma R (2014) Association mapping: a new paradigm for dissection of complex traits in crops. In: Kishor PBK, Bandopadhyay R, Suravajhala P (eds) Agricultural bioinformatics. Springer India, New Delhi, pp 1-20

R Development Core Team (2011) R: a language and environment for statistical computing. The R Foundation for Statistical Computing, Vienna. ISBN: 3-900051-07-0. http://www.R-project.org/. Accessed 7 Feb 2017

Rasheed A, Xia X, Ogbonnaya F, Mahmood T, Zhang Z, Mujeeb-Kazi A, He Z (2014) Genome-wide association for grain morphology in synthetic hexaploid wheats using digital imaging analysis. BMC Plant Biol 14:128

Reed E, Nunez S, Kulp D, Qian J, Reilly MP, Foulkes AS (2015) A guide to genome-wide association analysis and post-analytic interrogation. Stat Med 34:3769-3792

RiceData (2012) http://www.ricedata.cn/gene/gene_pi.htm. Accessed 22 Oct 2018

Sakai H, Lee SS, Tanaka T, Numa H, Kim J, Kawahara Y, Wakimoto H, Yang C, Iwamoto M, Abe T, Yamada Y, Muto A, Inokuchi H, Ikemura T, Matsumoto T, Sasaki T, Itoh T (2013) Rice Annotation Project Database (RAP-DB): an integrative and interactive database for rice genomics. Plant Cell Physiol 54:e6

Sharma TR, Rai AK, Gupta SK, Vijayan J, Devanna BN, Ray S (2012) Rice blast management through host-plant resistance: retrospect and prospects. Agric Res 1:37-52

Shirsekar G, Dai L, Hu Y, Wang X, Zeng L, Wang GL (2010) Role of ubiquitination in plant innate immunity and pathogen virulence. J Plant Biol 53:10-18

Soto-Cerda BJ, Cloutier S (2012) Association mapping in plant genomes. In: Caliskan M (ed) Genetic diversity in plants, 498p, 1st edn. InTech, Rijek, pp 29-54

Su J, Wang W, Han J, Chen S, Wang C, Zeng L, Feng A, Yang J, Zhou B, Zhu X (2015) Functional divergence of duplicated genes results in a novel blast resistance gene Pi50 at the Pi2/9 locus. Theor Appl Genet 128:2213-2225
Tang J, Zhu X, Wang Y, Liu L, Xu B, Li F, Fang J, Chu C (2011) Semi-dominant mutations in the CC-NB-LRR-type $R$ gene, NLS1, lead to constitutive activation of defense responses in rice. Plant J 66:996-1007

Valent B, Farrall L, Chumley FG (1991) Magnaporthe grisea genes for pathogenicity and virulence identified through a series of backcrosses. Genetics 127:87-101

Wan H, Chen L, Guo J, Li Q, Wen J, Yi B, Ma C, Tu J, Fu T, Shen J (2017) Genomewide association study reveals the genetic architecture underlying salt tolerance-related traits in rapeseed (Brassica napus L.). Front Plant Sci 8:593

Wang C, Yang Y, Yuan X, Xu Q, Feng Y, Yu H, Wang Y, Wei X (2014) Genome-wide association study of blast resistance in indica rice. BMC Plant Biol 14:311

Wilson RA, Talbot NJ (2009) Under pressure: investigating the biology of plant infection by Magnaporthe oryzae. Nat Rev Microbiol 7:185-195

Xu X, Hayashi N, Wang CT, Fukuoka S, Kawasaki S, Takatsuji H, Jiang CJ (2014) Rice blast resistance gene Pikahei-1(t), a member of a resistance gene cluster on chromosome 4, encodes a nucleotide-binding site and leucine-rich repeat protein. Mol Breed 34:691-700

Zeigler RS, Leong SA, Teng PS (1994) Rice blast disease, 1st edn. CAB International, Wallingford, p 626

Zhao K, Tung C-W, Eizenga GC, Wright MH, Ali ML, Price AH, Norton GJ, Islam MR, Reynolds A, Mezey J, McClung AM, Bustamante CD, McCouch SR (2011) Genome-wide association mapping reveals a rich genetic architecture of complex traits in Oryza sativa. Nat Commun 2:467

Zhao H, Wang X, Jia Y, Minkenberg B, Wheatley M, Fan J, Jia MH, Famoso A, Edwards JD, Wamishe Y, Valent B, Wang GL, Yang Y (2018) The rice blast resistance gene $P$ tr encodes an atypical protein required for broad-spectrum disease resistance. Nat Commun 9:2039

Zhu D, Kang H, Li Z, Liu M, Zhu X, Wang Y, Wang D, Wang Z, Liu W, Wang GL (2016) A genome-wide association study of field resistance to Magnaporthe oryzae in rice. Rice 9:44

\section{Submit your manuscript to a SpringerOpen ${ }^{\circ}$ journal and benefit from:}

- Convenient online submission

- Rigorous peer review

- Open access: articles freely available online

- High visibility within the field

- Retaining the copyright to your article

Submit your next manuscript at $\boldsymbol{\nabla}$ springeropen.com 